\title{
"Gestión de la ley 29719 que promueve la convivencia sin violencia y la convivencia escolar en la Institución Educativa Emblemática Luis fabio Xammar-Huacho 2017"
}

"Management of the law 29719 that promotes coexistence without violence and school coexistence in the Institutional educational Emblematic Luis Fabio Xammar-Huacho 2017"

\author{
Magaly Analí Galindo Kimura, Cristián Iván Escurra Estrada, Johnny Mitchell Gomero Mancesidor
}

\begin{abstract}
RESUMEN
Objetivo: Determinar la relación entre la gestión de la ley 29719 que promueve la convivencia sin violencia y la convivencia escolar en la Institución Educativa Emblemática "Luis Fabio Xammar Jurado", Santa María, 2017. Metodologia: La investigación realizada fue de enfoque cuantitativo, tipo básico de nivel descriptivo, diseño no experimental y correlacional de corte transversal. La población estuvo conformada por 120 docentes, la muestra fue de 60 docentes siendo el tipo de muestreo no probabilístico intencionado. Los datos se recolectaron haciendo uso de la técnica de la encuesta y se utilizó como instrumento un cuestionario para cada variable de estudio. Resultados los análisis estadístico refieren que existe relación positiva media $(r=0,430$, donde $p<$ de 0,05$)$ entre los niveles de la gestión de la Ley 29719 que promueve la convivencia sin violencia y la convivencia escolar. Conclusión: La gestión de la ley 29719 en la institución Luis Fabio Xamar Jurado; se relaciona en un nivel medio en relación alas relaciones interpersonales, normas institucionales, practica de valores y participacion estudiantil.
\end{abstract}

Palabras clave: Gestión, Ley 29719, convivencia, violencia.

\section{ABSTRACT}

Objective: To determine the relationship between the management of law 29719 that promotes coexistence without violence and school coexistence in the Emblematic Educational Institution "Luis Fabio Xammar Jurado", Santa María, 2017. Methodology: The research was quantitative approach , basic type of descriptive level, non-experimental design and cross-sectional correlational design. The population was made up of 120 teachers, the sample was 60 teachers being the type of non-probabilistic sampling intended. Data were collected using the survey technique and a questionnaire was used as an instrument for each study variable. Results The statistical analyzes indicate that there is a positive average relationship $(r=0.430$ where $p<0.05$ ) between the levels of the management of Law 29719 that promotes coexistence without violence and school coexistence. Conclusion: The management of law 29719 in the Luis Fabio Xamar Jurado institution; It is related at a medium level in relation to interpersonal relationships, institutional norms, values practice and student participation.
\end{abstract}

Keywords: Management, Law 29719, coexistence, violence.

\section{INTRODUCCIÓN}

La presente investigación trata acerca de la relación que existe entre Gestión de la ley 29719 que promueve la convivencia sin violencia y la convivencia escolar en la Institución Educativa Emblemática Luis Fabio XammarHuacho 2017. La variable Gestión de la ley 29719 se trabajó tomando en consideración 3 dimensiones y la variable convivencia escolar teniendo en cuenta 4 variables, la tesis se desarrolló teniendo en cuenta el esquema de la universidad. De los antecedentes podemos citar a Garretón (2013), en Estado de la convivencia escolar, conflictividad y su forma de abordarla en establecimientos educacionales de alta vulnerabilidad social de la Provincia de Concepción, Chile, se concluyó que las normas y su ejecución como característica de la percepción de la convivencia en un nivel de relación positiva. Carvajal (2012), en Convivencia escolar en adolescentes de cinco municipios de sabana centro departamento de Cundinamarca - Colombia, concluyó que según los estudiantes la mayor frecuencia de los conflictos está dado por estudiantes conflictivos, falta de respeto a la autoridad, falta de manejo disciplinario e intolerancia por parte de los profesores. Muñoz (2012), en Liceo de la comuna de Talca, Chile, con estudiantes de cuarto medio, resultados principales se observa que los jóvenes perciben que el liceo es altamente vulnerable a la violencia escolar; los varones la definen como normal y las damas la representan como negativa.

\section{Revisión de la Literatura}

Definición de la Ley 29719 que promueve la convivencia sin violencia

En concordancia con el reglamento de la ley № 29719 ley que promueve la convivencia sin violencia en las instituciones educativas, capítulo I disposiciones generales, se señaló que

Art. $1^{\circ}$ Del objeto: El presente reglamento tiene por objeto establecer el marco regulador para que las instituciones educativas garanticen condiciones adecuadas de convivencia democrática entre las y los estudiantes y los demás miembros de la comunidad educativa, y normen las medidas y procedimientos de protección y atención integral, ante casos de violencia y acoso entre las y los estudiantes; tomando en cuenta los diversos ámbitos culturales y bilingües. Art. $2^{\circ}$ De los principios, derechos y responsabilidades para la aplicación del presente Reglamento, se tendrá en cuenta los principios, derechos, deberes o responsabilidades contemplados en la Constitución Política del Perú, la Convención sobre los Derechos de los Niños; la Ley $N^{\circ} 28044$, Ley General de Educación y la Ley $N^{\circ} 27337$, que aprueba el Código de los Niños y Adolescentes. Art. $3^{\circ}$ De la Convivencia Democrática en la institución educativa La convivencia es el conjunto de relaciones interpersonales cotidianas, caracterizadas por el respeto y valoración del otro; construida y aprendida en la práctica cotidiana de la escuela entre los miembros de la comunidad educativa; favorece el desarrollo de competencias para la formación integral de las y los estudiantes, en un marco ético de respeto y ejercicio de los derechos humanos. Art. $4^{\circ}$ Finalidad de la Convivencia Democrática en la institución educativa La convivencia democrática en la institución educativa tiene como finalidad propiciar una cultura de paz como base para la formación integral de las y los estudiantes que los prepare para la vida y el ejercicio de su ciudadanía, promoviendo entornos acogedores e integradores, que contribuyan a climas institucionales favorables para su desarrollo integral. Art. $5^{\circ}$ Del ámbito de aplicación La Ley y el presente Reglamento, 
serán de aplicación en todas las instituciones y programas educativos públicos e instituciones educativas privadas de Educación Básica, Educación Técnico-Productiva e Instituciones de Educación Superior No Universitaria.

Tomando como referencia la definición de la ley 29719 que promueve la convivencia sin violencia o la también conocida ley antibullyng, es fundamental conocer el objeto de este reglamento pues garantiza se cumplan los ambientes idóneos para una atención integral de los estudiantes y miembros de la comunidad educativa, en cuanto a la convivencia escolar. En las instituciones de nuestro país se debería tener en claro los principios, derechos, deberes o responsabilidades que fundamentan la aplicación de este reglamento

El Minedu, en su manual de tutoría y orientación educativa (2013), nos dice sobre las funciones del equipo responsable de la convivencia democrática

Desde la Dirección de Tutoría y Orientación Educativa, definimos la Convivencia Democrática como el "conjunto de acciones organizadas caracterizadas por relaciones interpersonales democráticas entre todos los miembros de la comunidad educativa, que favorecen un estilo de vida ético y la formación integral de los estudiantes ".18 La implementación de la Convivencia Democrática promueve: La construcción de una convivencia escolar que garantice el ejercicio de los derechos, la democracia y ciudadanía. La elaboración de normas consensuadas. La participación de todas las instancias de la comunidad educativa y en particular la participación estudiantil. (p. 47)

De acuerdo con el reglamento de la Ley $N^{\circ} 29719$, ley que promueve la convivencia sin violencia en las instituciones educativas Artículo 11.- Funciones del director o directora de la institución educativa efectúa las siguientes funciones:

a)Garantizar la elaboración e implementación del Plan de Convivencia Democrática de la institución educativa.

b) Supervisar que los procedimientos y medidas correctivas se establezcan y ejecuten en el marco de la ley, el presente Reglamento y su correspondiente Directiva.

c)Apoyar las acciones del equipo responsable de la Convivencia Democrática en la institución educativa.

d)Comunicar y rendir cuenta acerca de los procesos y logros de la Convivencia Democrática a la asamblea de padres y madres de familia y a los demás integrantes de la comunidad educativa.

Minedu (s/f) a través del Vice Ministerio de Gestión Institucional señaló que

El Consejo Educativo Institucional es un órgano de participación, concertación y vigilancia ciudadana de la Institución Educativa, que contribuye a la promoción y ejercicio de una gestión educativa de calidad de carácter transparente, moral y democrático. Su naturaleza tiene que ver con la participación de los representantes de la comunidad que genera mecanismos idóneos para ejercer la vigilancia de la gestión del Centro, así como para atender los derechos de sus integrantes orientados a fortalecer las capacidades decisorias del director de la Institución Educativa. (p. 12).

De acuerdo con el Minedu (2013) en el documento sobre tutoría y orientación educativa, aprendiendo a resolver conflictos en las instituciones educativas, señala acerca de la convivencia escolar:

Las instituciones educativas son espacios de formación para el aprendizaje de la convivencia democrática. Ello requiere que constituyan espacios protectores y promotores del desarrollo, donde todos sus integrantes sean valorados, protegidos, respetados, tengan oportunidades para hacerse responsables de las consecuencias de sus actos y reafirmen su valoración personal. Veamos el siguiente ejemplo, y reflexionemos sobre cómo nuestro actuar cotidiano tiene influencia en la convivencia y el clima en la institución educativa. (p. 46).

\section{METODOLOGÍA}

La investigación fue de tipo básica con un nivel descriptivo. Este estudio fue correlacional, tal como lo manifestaron (Hernández Sampieri, Fernández collado, \& Baptista Lucio, 2014)al indicar que la investigación correlacional asocia variables mediante un patrón predecible para un grupo o población. (p. 81). Por el diseño, la presente investigación fue no experimental, ya que se basó en las observaciones de los hechos en estado natural, sin la intervención o manipulación del investigador. Del mismo modo, el diseño de investigación fue transversal. En la presente investigación la población de docentes fue de 60 con la que se trabajó, por lo que no se calculó la muestra. Para recopilar los datos de las variables en estudio se utilizó la técnica de la encuesta.

\section{RESULTADOS}

Tabla 1: Correlación entre los niveles de la gestión de la Ley 29719 que promueve la convivencia sin violencia y convivencia escolar

\begin{tabular}{|c|c|c|c|}
\hline$R$ de Pearson & & $\begin{array}{c}\text { niveles de la } \\
\text { gestión de la } \\
\text { Ley } 29719\end{array}$ & $\begin{array}{c}\text { convivencia } \\
\text { escolar }\end{array}$ \\
\hline \multirow{2}{*}{$\begin{array}{c}\text { niveles de la } \\
\text { gestión de la } \\
\text { Ley } \\
29719\end{array}$} & $\begin{array}{l}\text { Coeficiente de } \\
\text { correlación }\end{array}$ & 1,000 & 0,430 \\
\hline & Sig. (bilateral) & & 0,001 \\
\hline
\end{tabular}

60

60

Los resultados del análisis estadístico refieren una relación positiva media ( $r$ de pearson $=0,430$ donde $p<$ de 0,01 ) entre los niveles de la gestión de la Ley 29719 que promueve la convivencia sin violencia y la convivencia escolar en la I.E.E "Luis Fabio Xammar", distrito de Santa María, 2017. Al tenerse una significancia bilateral de 0,001 la misma que se encuentra muy por debajo del valor permitido $(0,01)$, se comprueba la hipótesis planteada en el sentido siguiente: Existe relación altamente significativa de manera positiva entre la gestión de la Ley 29719 que promueve la convivencia sin violencia y la convivencia escolar en la I.E.E "Luis Fabio Xammar", distrito de Santa María, 2017. Los resultados son al $99 \%$ de confianza.

\section{DISCUSIÓN}

Se observa que la gestión de la ley 29719 referida a los componentes funciones del equipo responsable, funciones de la directora, funciones del equipo institucional se relaciona en un nivel medio con la convivencia escolar en cuanto al conocimiento, ejecución y aplicación de las normas que son formativas, de prevención y de atención integral que se debería desarrollar en la institución educativa. Algunos antecedentes que se comparan con el estudio son, Carvajal. M (2012), en Convivencia escolar en adolescentes de cinco municipios de sabana centro departamento de Cundinamarca - Colombia, concluyó que según los estudiantes la mayor frecuencia de los conflictos está dado por estudiantes conflictivos, falta de respeto a la autoridad, falta de manejo disciplinario e intolerancia por parte de los profesores. En cuanto al componente de la gestión de la ley 
que promueve la convivencia sin violencia los resultados de la investigación reportan a un $95 \%$ de confianza una relación media $(r=0,498)$ entre el componente gestión del equipo responsable de convivencia democrática y la convivencia escolar en la I.E.E "Luis Fabio Xammar", distrito de Santa María, 2017. Esto indica que las funciones del equipo responsable en cuanto a planificar, implementar, ejecutar y evaluar el plan se relacionan medianamente con la convivencia escolar. Cuenca (2016), en "Inteligencia emocional y actitud hacia la conducta violenta en estudiantes de nivel secundario de una institución educativa del distrito de San Martín de Porres", se halló una relación media entre las dos variables propuestas. En cuanto al componente funciones de la gestión directiva de convivencia democrática los resultados de la investigación reportan a un $95 \%$ de confianza una relación moderada $(r=0,491)$ con la convivencia escolar en la I.E.E "Luis Fabio Xammar", distrito de Santa María, 2017. Es decir, que la planificación, organización, coordinación, dirección y evaluación de todas las actividades se relacionan medianamente con la convivencia escolar. Garretón (2013) en "Estado de la convivencia escolar, conflictividad y su forma de abordarla en establecimientos educacionales de alta vulnerabilidad social de la Provincia de Concepción, Chile", se concluye que las normas y su ejecución como característica de la percepción de la convivencia en positivo, son unos de los buenos elementos de la convivencia de estos centros, observándose en los resultados que los estudiantes perciben que las normas de convivencia son aplicadas sin mayores diferencias por sus docentes. Respecto al componente funciones de la gestión del consejo educativo institucional de la convivencia democrática, al 95\% de confianza se relaciona en un nivel bajo $(r=0,363)$ con la convivencia escolar en la I.E.E "Luis Fabio Xammar", distrito de Santa María, 2017. Esto es, que la implementación, ejecución y supervisión del plan que conforman el componente funciones del consejo educativo institucional se relacionan débilmente con la convivencia escolar.

\section{CONCLUSIONES}

Los resultados de la investigación dan cuenta de una relación media $(r=0,430$, donde $p<$ de 0,01$)$ entre los niveles de la gestión de la Ley 29719 que promueve la convivencia sin violencia y la convivencia escolar en la I.E.E "Luis Fabio Xammar", distrito de Santa María, 2017. Esto indica que la gestión de la ley 29719 que promueve la convivencia sin violencia referida a los componentes funciones del equipo responsable de la convivencia democrática, funciones del directora o directora y funciones del consejo educativo institucional se relacionan en un nivel medio con la convivencia escolar en cuanto a las relaciones interpersonales, normas institucionales, práctica de valores y la participación estudiantil.

\section{AGRADECIMIENTO}

Se le agradece a todos los docentes de la I.E.E "Luis Fabio Xammar" que colaboraron en el llenado de cuestionarios, que sirvieron para contrastar nuestras hipótesis planteadas.

\section{REFERENCIAS BIBLIOGRAFICAS}

Carvajal (2012). Convivencia escolar en adolescentes de cinco municipios de sabana centro departamento de Cundinamarca - Colombia.

Cuenca.R.N.E. (2016). Inteligencia emocional y actitud hacia la conducta violenta en estudiantes de nivel secundario de una institución educativa del distrito de San Martín de Porres. Tesis para optar grado de Doctor. Escuela de Pos grado. Universidad Femenina del Sagrado Corazón. Junin.

Congreso de la República (2011) Ley N²9719. Reglamento de ley $N^{\circ} 29719$, que promueve la convivencia sin violencia en las instituciones educativas.

Garretón, P. (2013). Estado de la convivencia escolar, conflictividad y su forma de

abordarla en establecimientos educacionales de alta vulnerabilidad social de la Provincia de Concepción, Chile. Recuperada desde:

http://helvia.uco.es/xmlui/bitstream/handle/10396/ 11611/2014000000906. pdf?sequence $=1$.

Hernández, R., Fernández C. C., \& Baptista L. M. (2014). Metodología de la Investigación. México: McGrawHill / Interamericana Editores, S.A. DE C.V.

Minedu (2012). Reglamento de ley $N^{\circ} 29719$, Lo que promueve la convivencia sin violencia en las instituciones educativas

Minedu (2013). Tutoría y orientación educativa. Recuperada desde:

http://www.minedu.gob.pe/minedu/archivos/a/002/ 02-bibliografia-comun-a-ebr-eba-y-etp/7aprendiendo-a-resolver-conflictos-en-lasinstituciones-educativas.pdf.

Muñoz, M. (2012) Percepciones y significados sobre la convivencia y violencia escolar de estudiantes de cuarto medio de un liceo municipal de Chile. Revista de Pedagogía, Vol. 28, № 82 Escuela de Educación Universidad Central de Venezuela Caracas, Mayo-Agosto de 2007, 197-224.

Vice Ministerio de Gestión Institucional (s/f). Manual del Consejo educativo Institucional. Recuperada desde:

https://es.scribd.com/doc/7703743/Manual-delConsejo-Educativo-Institucional 